Research Article

\title{
Solvability in Gevrey Classes of Some Nonlinear Fractional Functional Differential Equations
}

\author{
Hicham Zoubeir (D) \\ Ibn Tofail University, Department of Mathematics, Faculty of Sciences, P.O. Box 133, Kenitra, Morocco \\ Correspondence should be addressed to Hicham Zoubeir; hzoubeir2014@gmail.com
}

Received 11 August 2019; Accepted 28 October 2019; Published 29 June 2020

Academic Editor: Julio D. Rossi

Copyright (C) 2020 Hicham Zoubeir. This is an open access article distributed under the Creative Commons Attribution License, which permits unrestricted use, distribution, and reproduction in any medium, provided the original work is properly cited.

Our purpose in this paper is to prove, under some regularity conditions on the data, the solvability in a Gevrey class of bound -1 on the interval $[-1,1]$ of a class of nonlinear fractional functional differential equations.

\section{Introduction}

Fractional calculus has evolved from the speculations of early mathematicians of the $17^{\text {th }}$ and $18^{\text {th }}$ centuries like G. W. Leibnitz, I. Newton, L. Euler, G. F. de L'Hospital, and J. L. Lagrange [1]. In the $19^{\text {th }}$ century, other eminent mathematicians like P. S. Laplace, J. Liouville, B. Riemann, E. A. Holmgren, O. Heaviside, A. Grunwald, A. Letnikov, J. B. J. Fourier, and N. H. Abel have used the ideas of fractional calculus to solve some physical or mathematical problems [1]. In the $20^{\text {th }}$ century, several mathematicians (S. Pincherle, O. Heaviside, G. H. Hardy, H. Weyl, E. Post, T. J. Fa Bromwich, A. Zygmund, A. Erdelyi, R. G. Buschman, M. Caputo, etc.) have made considerable progress in their quest for rigor and generality to build fractional calculus and its applications on rigorous and solid mathematical foundations [1]. Actually, fractional calculus allows mathematical modeling of social and natural phenomena in a more powerful way than the classical calculus. Indeed fractional calculus has a lot of applications in different areas of pure and applied sciences like mathematics, physics, engineering, fractal phenomena, biology, social sciences, finance, economy, chemistry, anomalous diffusion, and rheology [1-22]. It is then of capital importance to develop for fractional calculus the mathematical tools analogous to those of classical calculus $[1,3,4,19,23]$. The fractional differential equations [23-28] are a particularly important case of such fundamental tools. An important type of fractional differential equations is that of fractional functional differential equations (FFDEs) [10, 29-31] which are the fractional analogues to functional differential equations $[17,32-34]$, enable the study of some physical, biological, social, and economical processes (automatic control, financial dynamics, economical planning, population dynamics, blood cell dynamics, infectious disease dynamics, etc.) with fractal memory and nonlocality effects, where the rate of change of the state of the systems depends not only on the present time but on other different times which are functions of the present time $[11,35,36]$. The question then arises of the choice of a suitable framework for the study of the solvability of these equations. But, since the functional Gevrey spaces play an important role in various branches of partial and ordinary differential equations [37-40], we think that these functional spaces can play the role of such convenient framework. However, let us point out that in order to make these spaces adequate to our specific setting, it is necessary to make a modification to their definition. This leads us to the definition of new Gevrey classes, namely, the Gevrey classes $G_{l, q_{1}}\left(\left[q_{1}, q_{2}\right]\right)$ of bound $q_{1}$ and index $l>0$ on an interval $\left[q_{1}, q_{2}\right]$. Our purpose in this paper is to prove, under some regularity conditions on the data, the solvability in a Gevrey class of the form $G_{k,-1}([-1,1])$ of a class of nonlinear FFDE. Our approach is mainly based on a theorem that we have proved in [41]. The notion of fractional calculus we are interested in is the Caputo fractional calculus. Some examples are given to illustrate our main results. 


\section{Preliminary Notes and Statement of the Main Result}

2.1. Basic Notations. Let $F: E \longrightarrow E$ be a mapping from a nonempty set $E$ into itself. $F^{\langle n\rangle}(n \in \mathbb{N})$ denotes the iterate of $F$ of order $n$ for the composition of mappings.

For $z \in \mathbb{C}$ and $h>0, B(z, h)$ is the open ball in $\mathbb{C} \simeq \mathbb{R}^{2}$ with the center $z$ and radius $h$.

Let $S_{1}$ and $S_{2}$ be two nonempty subsets of $\mathbb{C}$ such that $S_{1} \subset S_{2}$ and $f: S_{2} \longrightarrow \mathbb{C}$ a mapping. We denote by $f_{\mid S_{1}}$ the restriction of the mapping $f$ to the set $S_{1}$.

For $z \in \mathbb{C}$ and $S \subset \mathbb{C}(S$ nonempty $)$ we set

$$
\varrho(z, S):=\inf _{\zeta \in S}(|z-\zeta|) .
$$

For $l, \varphi, r>0$, and $n \in \mathbb{N}^{*}$, we set for every nontrivial compact interval $\left[q_{1}, q_{2}\right]$ of $\mathbb{R}$

$$
\left\{\begin{array}{l}
{\left[q_{1}, q_{2}\right]_{r}:=\left\{x+\zeta: x \in\left[q_{1}, q_{2}\right], \zeta \in B(0, r)\right\}} \\
{\left[q_{1}, q_{2}\right]_{l, r, n}:=\left[q_{1}, q_{2}\right]_{r n^{-1 / l}},} \\
{\left[q_{1}, q_{2}\right]^{\varphi, r}:=\left\{q_{1}+s e^{i \theta}: s \in\right] 0, q_{2}-q_{1}+r[, \theta \in]-\varphi, \varphi[\}} \\
{\left[q_{1}, q_{2}\right]^{r}:=\left[q_{1}, q_{2}\right]^{r, r}} \\
{\left[q_{1}, q_{2}\right]^{l, r, n}:=\left[q_{1}, q_{2}\right]^{r n^{-1 / l}} .}
\end{array}\right.
$$

Thus, we have

$$
\left\{\begin{array}{l}
{\left[q_{1}, q_{2}\right]_{r}=\left\{z \in \mathbb{C}: \varrho\left(z,\left[q_{1}, q_{2}\right]\right)<r\right\},} \\
{\left[q_{1}, q_{2}\right]_{l, r, n}=\left\{z \in \mathbb{C}: \varrho\left(z,\left[q_{1}, q_{2}\right]\right)<r n^{-1 / l}\right\} .}
\end{array}\right.
$$

Remark 1. The following inclusions hold for every $\left.\left.d \in] q_{1}, q_{2}\right], r \in\right] 0, d-q_{1}\left[\right.$ and $n \in \mathbb{N}^{*}$ :

$$
\left\{\begin{array}{l}
{\left[d, q_{2}\right]_{r} \subset\left[q_{1}, q_{2}\right]^{r},} \\
{\left[d, q_{2}\right]_{l, r, n} \subset\left[q_{1}, q_{2}\right]^{l, r, n} .}
\end{array}\right.
$$

Let $f: S \longrightarrow \mathbb{C}$ be a bounded function. $\|f\|_{\infty, S}$ denotes the quantity:

$$
\|f\|_{\infty, S}:=\sup _{z \in S}|f(s)|
$$

By $C^{0}\left(\left[q_{1}, q_{2}\right]\right)$ (resp. $\left.C^{1}\left(\left[q_{1}, q_{2}\right]\right)\right)$, we denote the complex vector space of all complex valued functions defined and continuous (resp. defined and of class $C^{1}$ ) on the interval $\left[q_{1}, q_{2}\right] . C^{0}\left(\left[q_{1}, q_{2}\right]\right)$ is a Banach space when it is endowed with the uniform norm:

$$
\|\cdot\|_{\infty,\left[q_{1}, q_{2}\right]}: f \in C^{0}\left(\left[q_{1}, q_{2}\right]\right) \longmapsto\|f\|_{\infty,\left[q_{1}, q_{2}\right]} .
$$

For every $r \geq 0$, we denote by $\bar{\Delta}_{\infty}(r)$ the closed ball in $\left.C^{0}([-1,1])\right)$ of radius $r$ and center, the null function.

Let $\xi_{1}, \xi_{2} \in \mathbb{C}$. We denote by $\xi_{1}, \xi_{2}$ the linear path joining $\xi_{1}$ to $\xi_{2}$ :

$$
\begin{aligned}
\stackrel{\xi_{1}, \xi_{2}}{\longrightarrow}:[0,1] & \longrightarrow \mathbb{C}, \\
t & \longmapsto(1-t) \xi_{1}+t \xi_{2} .
\end{aligned}
$$

In this paper, $k>0$ and $\alpha \in] 0,1[$ are fixed numbers.

\subsection{Fractional Derivatives and Integrals}

Definition 1. Let $\delta \in] 0,1$ [ and $f$ be a Lebesgue-integrable function on the nontrivial compact interval $\left[q_{1}, q_{2}\right]$. The Caputo fractional integral of order $\delta$ and lower bound $q_{1}$ of the function $f[19,23,25,26,28]$ is the function denoted by ${ }^{c} I_{a}^{\delta} f$ and defined by

$$
{ }^{c} I_{q_{1}}^{\delta} f(t):=\frac{1}{\Gamma(\delta)} \int_{q_{1}}^{t}(t-s)^{\delta-1} f(s) \mathrm{d} s, \quad t \in\left[q_{1}, q_{2}\right],
$$

where $\Gamma$ denotes the classical gamma function.

Remark 2. If the function $f$ is continuous on the interval $\left[q_{1}, q_{2}\right]$, then the function ${ }^{c} I_{q_{1}}^{\delta} f$ is well defined and continuous on the entire interval $\left[q_{1}, q_{2}\right]$, and we have

$$
{ }^{c} I_{q_{1}}^{\delta} f\left(q_{1}\right)=0 \text {. }
$$

Definition 2. Let $f:\left[q_{1}, q_{2}\right] \longrightarrow \mathbb{C}$ be an absolutely continuous function on $\left[q_{1}, q_{2}\right]$; then, the Caputo fractional derivative of $f$ of order $\delta$ and lower bound $q_{1}$ $[19,23,25,26,28]$ is the function denoted by ${ }^{c} D_{q_{1}}^{\delta} f$ and defined by

$$
{ }^{c} D_{q_{1}}^{\delta} f(t):=\frac{1}{\Gamma(1-\delta)} \int_{q_{1}}^{t}(t-s)^{-\delta} f^{\prime}(s) \mathrm{d} s, \quad t \in\left[q_{1}, q_{2}\right] .
$$

Remark 3. Let $f \in C^{1}\left(\left[q_{1}, q_{2}\right]\right)$. We have for every $x \in\left[q_{1}, q_{2}\right]$

$$
\left({ }^{c} I_{q_{1}}^{\delta} \circ{ }^{c} D_{q_{1}}^{\delta}\right) f(x)=f(x)-f\left(q_{1}\right) .
$$

If $f\left(q_{1}\right)=0$, then the Caputo fractional integral of the function $f$ of order $\delta,{ }^{c} I_{q_{1}}^{\delta} f$, is also of class $C^{1}$ on the interval $\left[q_{1}, q_{2}\right]$ and we have $[19,23,25,26,28]$

$$
\left({ }^{c} D_{q_{1}}^{\delta}{ }^{\circ} I_{q_{1}}^{\delta}\right) f=f
$$

\subsection{Gevrey Classes}

Definition 3. Let $l>0$. The Gevrey class of index $l$ on $\left[q_{1}, q_{2}\right]$, denoted by $G_{l}\left(\left[q_{1}, q_{2}\right]\right)$, is the set of all functions $f$ of class $C^{\infty}$ on $\left[q_{1}, q_{2}\right]$ such that

$$
\left\|f^{(n)}\right\|_{\infty,\left[q_{1}, q_{2}\right]} \leq B^{n+1} n^{n(1+(1 / l))}, \quad n \in \mathbb{N},
$$

where $B>0$ is a constant (with the convention that $0^{0}=1$ ).

Definition 4. The Gevrey class of bound $q_{1}$ and index $l$ on the interval $\left[q_{1}, q_{2}\right]$, denoted by $G_{l, q_{1}}\left(\left[q_{1}, q_{2}\right]\right)$, is the set of all functions $f$ of class $C^{1}$ on $\left[q_{1}, q_{2}\right]$ and of class $C^{\infty}$ on $\left.] q_{1}, q_{2}\right]$ such that the restriction $f_{\mid\left[q, q_{2}\right]}$ of $f$ belongs to the Gevrey class $G_{l}\left(\left[q, q_{2}\right]\right)$, for every $\left.q \in\right] q_{1}, q_{2}[$. 


\subsection{The Property $S(l)$}

Definition 5. A function $\varphi$ defined on the set $\left\{q_{1}\right\}$ $\cup\left[q_{1}, q_{2}\right]^{r}(r \in] 0, \pi[)$ is said to satisfy the property $S(l)$ on the interval $\left[q_{1}, q_{2}\right]$ if $\varphi_{\mid\left[q_{1}, q_{2}\right]^{r}}$ is holomorphic on $\left[q_{1}, q_{2}\right]^{r}, \varphi_{\mid\left[q_{1}, q_{2}\right]}$ is a function of class $C^{1}$ on $\left[q_{1}, q_{2}\right]$, and there exists a constant $\left.\tau_{\varphi} \in\right] 0, \pi[$ such that for all $D \in] 0, \tau_{\varphi}$ ] there exist $N_{l, \varphi}(D) \in \mathbb{N}^{*}$ depending only on $D, l$, and $\varphi$ such that the inclusion

$$
\varphi\left(\left[q_{1}, q_{2}\right]^{l, D, n+1}\right) \subset\left[q_{1}, q_{2}\right]^{l, D, n}
$$

holds for every integer $n \geq N_{l, \varphi}(D)$. The number $\tau_{\varphi}$ is then called a $S(l)$-threshold for the function $\varphi$.

Remark 4. Let $\varphi$ be a function verifying the property $S(l)$. Then,

$$
\varphi\left(\left[q_{1}, q_{2}\right]\right) \subset\left[q_{1}, q_{2}\right] .
$$

On the other hand, it follows from (14) that we have for every $D \in] 0, \tau_{\varphi}[$

$$
\varphi\left(\left[q_{1}, q_{2}\right]^{l, D, N_{l, \varphi}(D)(p+1)}\right) \subset\left[q_{1}, q_{2}\right]^{l, A, N_{l, \varphi}(D) p}, \quad p \in \mathbb{N}^{*} .
$$

Thence, we have

$\varphi\left(\left[q_{1}, q_{2}\right]^{l, D N_{l, \varphi}(D)^{-1 / l}, p+1}\right) \subset\left[q_{1}, q_{2}\right]^{l, D N_{l, \varphi}(D)^{-1 / l}, p}, \quad p \in \mathbb{N}^{*}$.

It follows that for every $D \in] 0, \tau_{\varphi}$ [ there exists $\left.E \in\right] 0, D[$ such that

$$
\varphi\left(\left[q_{1}, q_{2}\right]^{l, E, p+1}\right) \subset\left[q_{1}, q_{2}\right]^{l, E, p}, \quad p \in \mathbb{N}^{*}
$$

2.5. Statement of the Main Result. Our main result in this paper is the following.

Theorem 1. Let $\lambda \in \mathbb{C}$ and $\sigma>0$. Let $a, b$, and $\psi$ be holomorphic functions on $[-1,1]_{\sigma}$ and $\Phi$ be an entire function. We assume that the function $a$ is not identically vanishing and that there exist constants $\alpha_{0}, \beta_{0}>0$ such that

$$
|\Phi(z)| \leq \alpha_{0} e^{\beta_{0}|z|}, \quad z \in \mathbb{C}
$$

and that $\psi$ satisfies the property $S(k)$. We also assume that the following conditions are fulfilled:

$$
\begin{gathered}
a(-1)=b(-1)=0, \\
\frac{2^{\alpha}}{\alpha \Gamma(\alpha)}\|b\|_{\infty,[-1,1]}+|\lambda|<\frac{\ln \left(\alpha \Gamma(\alpha) / e \alpha_{0} \beta_{0} 2^{\alpha}\|a\|_{\infty,[-1,1]}\right)}{\beta_{0}},
\end{gathered}
$$

$$
\|a\|_{\infty,[-1,1]}\left\|\Phi^{\prime}\right\|_{\infty,\left[-\ln \left(\alpha \Gamma(\alpha) / \alpha_{0} \beta_{0}\|a\|_{\infty,[-1,1]}\right) / \beta_{0}, \ln \left(\alpha \Gamma(\alpha) / \alpha_{0} \beta_{0}\|a\|_{\infty,[-1,1]}\right) / \beta_{0}\right]}<\frac{\alpha \Gamma(\alpha)}{2^{\alpha}},
$$

$$
\left\|\psi^{\prime}\right\|_{\infty,[-1,1]} \leq 1+\alpha .
$$

Then, the FFDE

$$
(E):{ }^{c} D_{-1}^{\alpha} y(t)=a(t) \Phi(y(\psi(t)))+b(t)
$$

has a solution $u$ which belongs to the Gevrey class $G_{k,-1}([-1,1])$ and verifies the initial condition

$$
\left(E_{1}\right): y(-1)=\lambda \text {. }
$$

\section{Proof of the Main Result}

The proof of the theorem is subdivided in three steps.
Step 1. The localisation of the solutions of the equation:

$$
(\mathfrak{\Im}): r=\frac{\alpha_{0} 2^{\alpha}}{\alpha \Gamma(\alpha)}\|a\|_{\infty,[-1,1]} e^{\beta_{0} r}+\frac{2^{\alpha}}{\alpha \Gamma(\alpha)}\|b\|_{\infty,[-1,1]}+|\lambda| .
$$

The study of the variations of the function

$$
H: t \longmapsto \frac{\alpha_{0} 2^{\alpha}}{\alpha \Gamma(\alpha)}\|a\|_{\infty,[-1,1]} e^{\beta_{0} t}+\frac{2^{\alpha}}{\alpha \Gamma(\alpha)}\|b\|_{\infty,[-1,1]}+|\lambda|-t
$$

shows, under condition (21), that $H$ is strictly decreasing on $\left[0, \ln \left(\alpha \Gamma(\alpha) / \alpha_{0} \beta_{0} 2^{\alpha}\|a\|_{\infty,[-1,1]}\right) / \beta_{0}\right]$ and strictly increasing on $\left[\ln \left(\alpha \Gamma(\alpha) / \alpha_{0} \beta_{0} 2^{\alpha}\|a\|_{\infty,[-1,1]}\right) / \beta_{0},+\infty[\right.$. But, 


$$
\begin{aligned}
H(0) & =\frac{\alpha_{0} 2^{\alpha}}{\alpha \Gamma(\alpha)}\|a\|_{\infty,[-1,1]}+\frac{2^{\alpha}}{\alpha \Gamma(\alpha)}\|b\|_{\infty,[-1,1]}+|\lambda|>0, \\
H\left(\frac{\ln \left(\alpha \Gamma(\alpha) / \alpha_{0} \beta_{0} 2^{\alpha}\|a\|_{\infty,[-1,1]}\right)}{\beta_{0}}\right) & \\
= & \frac{2^{\alpha}}{\alpha \Gamma(\alpha)}\|b\|_{\infty,[-1,1]}+|\lambda| \\
& -\frac{\ln \left(\alpha \Gamma(\alpha) / e \alpha_{0} \beta_{0} 2^{\alpha}\|a\|_{\infty,[-1,1]}\right)}{\beta_{0}}<0 .
\end{aligned}
$$

Therefore, the equation $(\mathfrak{I})$ has on $\mathbb{R}^{+}$exactly two solutions $R_{0}<R_{1}$ and the following inequalities hold:

$$
0<R_{0}<\frac{\ln \left(\alpha \Gamma(\alpha) / \alpha_{0} \beta_{0} 2^{\alpha}\|a\|_{\infty,[-1,1]}\right)}{\beta_{0}}<R_{1} .
$$

Step 2. Proof of the existence of a solution $u$ of the FFDE $(E)$ in $C^{1}([-1,1])$ such that the initial condition $\left(E_{1}\right)$ holds.

Consider the operator $T: C^{0}([-1,1]) \longrightarrow C^{0}([-1,1])$ defined by the following formula:

$$
T(f)(t)={ }^{c} I_{-1}^{\alpha}(a .(\Phi \circ f \circ \psi)+b)(t)+\lambda, \quad t \in[-1,1] .
$$

We have for all $f \in \bar{\Delta}_{\infty}\left(R_{0}\right)$

$$
\begin{aligned}
& \|T(f)\|_{\infty,[-1,1]} \\
& \quad \leq\left\|{ }^{c} I_{-1}^{\alpha}(a .(\Phi \circ f \circ \psi)+b)\right\|_{\infty,[-1,1]}+|\lambda| \\
& \quad \leq \frac{2^{\alpha}}{\alpha \Gamma(\alpha)}\|a\|_{\infty,[-1,1]}\|\Phi \circ f\|_{\infty,[-1,1]}+\frac{2^{\alpha}}{\alpha \Gamma(\alpha)}\|b\|_{\infty,[-1,1]}+|\lambda| \\
& \quad \leq \frac{2^{\alpha}}{\alpha \Gamma(\alpha)}\|a\|_{\infty,[-1,1]} \alpha_{0} e^{\beta_{0}\|f\|_{\infty,[-1,1]}}+\frac{2^{\alpha}}{\alpha \Gamma(\alpha)}\|b\|_{\infty,[-1,1]}+|\lambda| \\
& \quad \leq \frac{\alpha_{0} 2^{\alpha}}{\alpha \Gamma(\alpha)}\|a\|_{\infty,[-1,1]} e^{\beta R_{0}}+\frac{2^{\alpha}}{\alpha \Gamma(\alpha)}\|b\|_{\infty,[-1,1]}+|\lambda|=R_{0} .
\end{aligned}
$$

Thence, the closed ball $\bar{\Delta}_{\mathrm{\infty}}\left(R_{0}\right)$ is stable by the operator $T$. On the other hand, we have for all $f, g \in \bar{\Delta}_{\infty}\left(R_{0}\right)$

$$
\begin{aligned}
& \|T(f)-T(g)\|_{\infty,[-1,1]} \\
& \quad \leq \frac{2^{\alpha}}{\alpha \Gamma(\alpha)}\|a\|_{\infty,[-1,1]}\left\|\Phi^{\prime}\right\|_{\infty,\left[-R_{0}, R_{0}\right]}\|f-g\|_{\infty,[-1,1]} .
\end{aligned}
$$

Since $0<R_{0}<\left(\ln \left(\alpha \Gamma(\alpha) / \alpha_{0} \beta_{0} 2^{\alpha}\|a\|_{\infty,[-1,1]}\right) / \beta_{0}\right)$, it follows from condition (23) that

$$
\frac{2^{\alpha}}{\alpha \Gamma(\alpha)}\|a\|_{\infty,[-1,1]}\left\|\Phi^{\prime}\right\|_{\infty,\left[-R_{0}, R_{0}\right]}<1
$$

Thence, $T$ has, in $\bar{\Delta}_{\infty}\left(R_{0}\right)$, a unique fixed point $u$.

Consider the sequence of functions $\left(f_{n}\right)_{n \in \mathbb{N}}$ defined on $[-1,1]$ by the following formula:

$$
f_{n}:=T^{\langle n\rangle}\left(f_{0}\right), \quad n \in \mathbb{N},
$$

where $f_{0}$ is the null function. Direct computations show that the functions $f_{n}$ belonging to $\bar{\Delta}_{\infty}\left(R_{0}\right)$ are of class $C^{1}$ on $[-1,1]$ and verify the following inequality:

$$
\left\|f_{n+1}-f_{n}\right\|_{\infty,[-1,1]} \leq\left\|f_{1}\right\|_{\infty,[-1,1]} Q^{n}, \quad n \in \mathbb{N}
$$

where

$$
Q:=\frac{2^{\alpha}}{\alpha \Gamma(\alpha)}\|a\|_{\infty,[-1,1]}\left\|\Phi^{\prime}\right\|_{\infty,\left[-R_{0}, R_{0}\right]} .
$$

Let us set for each $n \in \mathbb{N}, F_{n}:=f_{n+1}-f_{n}$. Since $Q \in\left[0,1\left[\right.\right.$, it follows that the function series $\sum F_{n}$ is uniformly convergent on $[-1,1]$ to a function $v \in \bar{\Delta}_{\infty}\left(R_{0}\right)$ which is a fixed point of the operator $T$. It follows that $v=u$. Consequently, the function series $\sum F_{n}$ is uniformly convergent on $[-1,1]$ to the function $u \in C^{0}([-1,1])$.

On the other hand, we have for all $x \in]-1,1]$ and $n \in \mathbb{N}^{*}:$

$$
\begin{aligned}
& F_{n+1}^{\prime}(x) \\
& =\frac{\alpha(x+1)^{\alpha-1}}{\Gamma(\alpha)} \int_{0}^{1}(1-t)^{\alpha-1} a(-1+t(x+1)) \cdot\left(\Phi\left(f_{n}(\psi(-1+t(x+1)))\right)-\Phi\left(f_{n-1}(\psi(-1+t(x+1)))\right)\right) \mathrm{d} t \\
& \quad+\frac{(x+1)^{\alpha}}{\Gamma(\alpha)} \int_{0}^{1}(1-t)^{\alpha-1} t a^{\prime}(-1+t(x+1)) \cdot\left(\Phi\left(f_{n}(\psi(-1+t(x+1)))\right)-\Phi\left(f_{n-1}(\psi(-1+t(x+1)))\right)\right) \mathrm{d} t \\
& \quad+\frac{(x+1)^{\alpha}}{\Gamma(\alpha)} \int_{0}^{1}(1-t)^{\alpha-1} t a(-1+t(x+1)) \psi^{\prime}(-1+t(x+1)) \cdot\left(\left(\Phi^{\prime}\left(f_{n}(\psi(-1+t(x+1)))\right)-\Phi^{\prime}\left(f_{n-1}(\psi(-1+t(x+1)))\right)\right)\right. \\
& \quad \cdot f_{n}^{\prime}(\psi(-1+t(x+1)))+\Phi^{\prime}\left(f_{n-1}(\psi(-1+t(x+1)))\right) \cdot\left(f_{n}^{\prime}(\psi(-1+t(x+1)))-f_{n-1}^{\prime}(\psi(-1+t(x+1)))\right) \mathrm{d} t .
\end{aligned}
$$


Since $a(-1)=0$, it follows that

$$
\begin{aligned}
\left\|F_{n+1}^{\prime}\right\|_{\infty,[-1,1]} & \frac{2^{\alpha}}{(\alpha+1) \Gamma(\alpha)}\left\|a^{\prime}\right\|_{\infty,[-1,1]}\left\|\Phi^{\prime}\right\|_{\infty,\left[-R_{0}, R_{0}\right]}\left\|F_{n}\right\|_{\infty,[-1,1]} \\
& +\frac{2^{\alpha}}{\alpha(\alpha+1) \Gamma(\alpha)}\left\|a^{\prime}\right\|_{\infty,[-1,1]}\left\|\Phi^{\prime}\right\|_{\infty,\left[-R_{0}, R_{0}\right]}\left\|F_{n}\right\|_{\infty,[-1,1]} \\
& +\frac{2^{\alpha}}{\alpha(\alpha+1) \Gamma(\alpha)}\left\|\psi^{\prime}\right\|_{\infty,[-1,1]}\|a\|_{\infty,[-1,1]}\left\|\Phi^{\prime \prime}\right\|_{\infty,\left[-R_{0}, R_{0}\right]}\left\|f_{n}^{\prime}\right\|_{\infty,[-1,1]}\left\|F_{n}\right\|_{\infty,[-1,1]} \\
& +\frac{2^{\alpha}}{\alpha(\alpha+1) \Gamma(\alpha)}\left\|\psi^{\prime}\right\|_{\infty,[-1,1]}\|a\|_{\infty,[-1,1]}\left\|\Phi^{\prime}\right\|_{\infty,\left[-R_{0}, R_{0}\right]}\left\|F_{n}^{\prime}\right\|_{\infty,[-1,1]} \\
\leq & \frac{2^{\alpha}}{\alpha \Gamma(\alpha)}\left\|a^{\prime}\right\|_{\infty,[-1,1]}\left\|\Phi^{\prime}\right\|_{\infty,\left[-R_{0}, R_{0}\right]}\left\|f_{1}\right\|_{\infty,[-1,1]} Q^{n} \\
& +\frac{2^{\alpha}}{\alpha \Gamma(\alpha)}\|a\|_{\infty,[-1,1]}\left\|\Phi^{\prime \prime}\right\|_{\infty,\left[-R_{0}, R_{0}\right]}\left\|f_{n}^{\prime}\right\|_{\infty,[-1,1]}\left\|f_{1}\right\|_{\infty,[-1,1]} Q^{n} \\
& +\frac{2^{\alpha}}{\alpha \Gamma(\alpha)}\|a\|_{\infty,[-1,1]}\left\|\Phi^{\prime}\right\|_{\infty,\left[-R_{0}, R_{0}\right]}\left\|F_{n}^{\prime}\right\|_{\infty,[-1,1]} .
\end{aligned}
$$

To achieve the proof of this step we need the following

Proof. We have for all $x \in]-1,1]$ and $n \in \mathbb{N}^{*}$ result.

Proposition 1. The sequence $\left(\left\|f_{n}^{\prime}\right\|_{\infty,[-1,1]}\right)_{n \in \mathbb{N}^{*}}$ is bounded.

$$
\begin{aligned}
f_{n+1}^{\prime}(x)= & \frac{\alpha(x+1)^{\alpha-1}}{\Gamma(\alpha)} \int_{0}^{1}(1-s)^{\alpha-1} \cdot\left(a(-1+s(x+1)) \cdot \Phi\left(f_{n}(\psi(-1+s(x+1)))\right)+b(-1+s(x+1))\right) \mathrm{d} s \\
& +\frac{(x+1)^{\alpha}}{\Gamma(\alpha)} \int_{0}^{1}(1-s)^{\alpha-1} s \cdot\left(a^{\prime}(-1+s(x+1)) \cdot \Phi\left(f_{n}(\psi(-1+s(x+1)))\right)+b^{\prime}(-1+s(x+1))\right) \mathrm{d} s \\
& +\frac{(x+1)^{\alpha}}{\Gamma(\alpha)} \int_{0}^{1}(1-s)^{\alpha-1} s a(-1+s(x+1)) \cdot \psi^{\prime}(-1+s(x+1)) \Phi^{\prime}\left(f_{n}(\psi(-1+s(x+1)))\right) \cdot f_{n}^{\prime}(\psi(-1+s(x+1))) \mathrm{d} s .
\end{aligned}
$$

It follows from assumption (20) that

$$
\begin{aligned}
\left\|f_{n+1}^{\prime}\right\|_{\infty,[-1,1]} \leq & \frac{2^{\alpha}}{\alpha \Gamma(\alpha)}\left(\left\|a^{\prime}\right\|_{\infty,[-1,1]}\|\Phi\|_{\infty,\left[-R_{0}, R_{0}\right]}+\left\|b^{\prime}\right\|_{\infty,[-1,1]}\right) \\
& +\frac{2^{\alpha}}{\alpha(\alpha+1) \Gamma(\alpha)}\|a\|_{\infty,[-1,1]} \cdot\left\|\psi^{\prime}\right\|_{\infty,[-1,1]} \\
& \cdot\left\|\Phi^{\prime}\right\|_{\infty,\left[-R_{0}, R_{0}\right]}\left\|f_{n}^{\prime}\right\|_{\infty,[-1,1]} .
\end{aligned}
$$

But, according to assumption (24) and (35) we have $\frac{2^{\alpha}}{\alpha(\alpha+1) \Gamma(\alpha)}\|a\|_{\infty,[-1,1]}\left\|\psi^{\prime}\right\|_{\infty,[-1,1]}\left\|\Phi^{\prime}\right\|_{\infty,\left[-R_{0}, R_{0}\right]} \leq \mathrm{Q}<1$.
Consequently, the following inequality holds for each $n \in \mathbb{N}^{*}$

$$
\begin{aligned}
\left\|f_{n+1}^{\prime}\right\|_{\infty,[-1,1]} \leq & \frac{2^{\alpha}}{\alpha \Gamma(\alpha)}\left(\left\|a^{\prime}\right\|_{\infty,[-1,1]}\|\Phi\|_{\infty,\left[-R_{0}, R_{0}\right]}+\left\|b^{\prime}\right\|_{\infty,[-1,1]}\right) \\
& +Q\left\|f_{n}^{\prime}\right\|_{\infty,[-1,1]} .
\end{aligned}
$$

Since $Q \in[0,1[$, it follows that the sequence $\left(\left\|f_{n}^{\prime}\right\|_{\infty,[-1,1]}\right)_{n \in \mathbb{N}^{*}}$ is bounded.

The proof of the proposition is complete.

Now, we set 


$$
\begin{aligned}
\theta:= & \left\|f_{1}\right\|_{\infty,[-1,1]}\left(\frac{2^{\alpha}}{\alpha \Gamma(\alpha)}\left\|a^{\prime}\right\|_{\infty,[-1,1]}\left\|\Phi^{\prime}\right\|_{\infty,\left[-R_{0}, R_{0}\right]}\right. \\
+ & \frac{2^{\alpha}}{\alpha \Gamma(\alpha)}\|a\|_{\infty,[1,1]}\left\|\Phi^{\prime \prime}\right\|_{\infty,\left[-R_{0}, R_{0}\right]} \\
& \left.\cdot \sup _{n \in \mathbb{N}^{*}}\left\|f_{n}^{\prime}\right\|_{\infty,[-1,1]}\right) .
\end{aligned}
$$

Then, we can write

$$
\left\|F_{n+1}^{\prime}\right\|_{\infty,[-1,1]} \leq \theta Q^{n}+Q\left\|F_{n}^{\prime}\right\|_{\infty,[-1,1]}, \quad n \in \mathbb{N}^{*} \text {. }
$$

Direct computations show then that

$$
\left\|F_{n+1}^{\prime}\right\|_{\infty,[-1,1]} \leq \theta n Q^{n}+\left\|F_{1}^{\prime}\right\|_{\infty,[-1,1]} Q^{n}, \quad n \in \mathbb{N}^{*} \text {. }
$$

Since $Q \in\left[0,1\left[\right.\right.$, it follows that the function series $\sum F_{n}^{\prime}$ is uniformly convergent on $[-1,1]$. Thence, the function $u$ is of class $C^{1}$ on $[-1,1]$ and satisfies the following relation:

$$
{ }^{c} I_{-1}^{\alpha}(a .(\Phi \circ u \circ \psi)+b)+\lambda=u .
$$

Consequently, according to assumption (20), we can write for all $t \in[-1,1]$

$$
\begin{array}{r}
{ }^{c} D_{-1}^{\alpha} u(t)={ }^{c} D_{-1}^{\alpha}\left[{ }^{c} I_{-1}^{\alpha}(a .(\Phi \circ u \circ \psi)+b)\right](t) \\
=a(t) \Phi(u(\psi(t)))+b(t) .
\end{array}
$$

So, $u$ is a solution of the FFDE $(E)$ which belongs to $C^{1}([-1,1])$ and fulfills the relation $u(-1)=\lambda$.

Step 3. Proof that $u$ belongs to the Gevrey class $G_{k,-1}([-1,1])$.

Since the function $\Lambda$ defined on $[0, \min (1, \sigma)[$ by $\Lambda:[0, \min (1, \sigma)[\longrightarrow \mathbb{R}$,

$$
\begin{aligned}
0 & \longmapsto \frac{2^{\alpha}}{\alpha \Gamma(\alpha)} \max \left(\begin{array}{c}
\|a\|_{\infty,[-1,1]}\left\|\Phi^{\prime}\right\|_{\infty,\left[-R_{0}, R_{0}\right]} \\
\|a\|_{\infty,[-1,1]}\|\Phi\|_{\infty,\left[-R_{0}, R_{0}\right]}+\|b\|_{\infty,[-1,1]}
\end{array}\right), \\
s>0 & \longmapsto \frac{(2+s)^{\alpha}}{\alpha \Gamma(\alpha)} \max \left(\begin{array}{c}
\|a\|_{\infty,[-1,1]^{s}}\left\|\Phi^{\prime}\right\|_{\infty,\left[-R_{0}, R_{0}\right]_{s}} \\
\|a\|_{\infty,[-1,1]^{s}}\|\Phi\|_{\infty,\left[-R_{0}, R_{0}\right]_{s}}+\|b\|_{\infty,[-1,1]^{s}}
\end{array}\right)
\end{aligned}
$$

is continuous on $[0, \min (1, \sigma)[$ and verifies by virtue of assumptions (22) and (23), the inequality $\Lambda(0)<1$. It follows that there exists $\left.s_{1} \in\right] 0, \min \left(1, \sigma, \tau_{\psi}\right)$ [ such that

$$
\Lambda\left(\left[0, s_{1}\right]\right) \subset[0,1[
$$

where $\tau_{\psi}$ is a $S(l)$-threshold of $\psi$. Let $d$ be an arbitrary but fixed element of ]-1,1[. Thanks to remark 4 , there exists $\left.s_{2} \in\right] 0, s_{1}$ [ such that the functions $a$ and $b$ are both holomorphic on $[-1,1]^{s_{2}}$ and the following condition holds:

$$
\psi\left([-1,1]^{k, s_{2}, n+1}\right) \subset[-1,1]^{k, s_{2, n}}, \quad n \in \mathbb{N}^{*} .
$$

Consider the sequence of functions $\left(\omega_{n}:[-1,1]^{k, s_{2}, n}\right.$ $\longrightarrow \mathbb{C})_{n \in \mathbb{N}^{*}}$, where

$$
\begin{aligned}
\omega_{1}(z) & =0, \text { for all } z \in[-1,1]^{k, s_{2}, 1}, \\
\omega_{n+1}(z) & =\frac{(z+1)^{\alpha}}{\Gamma(\alpha)} \int_{0}^{1}(1-s)^{\alpha-1} \\
& \left(\begin{array}{c}
a(-1+s(z+1)) \Phi\left(\omega_{n}(\psi(-1+s(z+1)))\right)+ \\
+b(-1+s(z+1))
\end{array}\right) \mathrm{d} s+\lambda,
\end{aligned}
$$

for each $n \in \mathbb{N}^{*}$ and $z \in[-1,1]^{k, s_{2}, n}$. Then, direct computations, based on (52), show that the function $\omega_{n}$ is for every $n \in \mathbb{N}^{*}$ holomorphic on $[-1,1]^{k, s_{2}, n}$.

Proposition 2. The inclusion $\omega_{n}\left([-1,1]^{k, s_{2}, n}\right) \subset\left[-R_{0}\right.$, $\left.R_{0}\right]_{k, s_{2}, n}$ holds for every $n \in \mathbb{N}^{*}$.

Proof. We denote the last inclusion by $P(n)$. We denote for every $z \in \mathbb{C}$ by $\widehat{z}$ the closest point of $[-1,1]$ to $z$. It is obvious that $P(1)$ is true. Assume for a certain $n \in \mathbb{N}^{*}$ that $P(p)$ is true for every $p \in\{1, \ldots, n\}$. Since the function $\omega_{n+1}$ is holomorphic on $[-1,1]^{k, s_{2}, n+1}$, we have then for each $z \in[-1,1]^{k, s_{2}, n+1}$

$$
\begin{aligned}
& \varrho\left(\omega_{n+1}(z),\left[-R_{0}, R_{0}\right]\right) \\
& \quad \leq\left|\omega_{n+1}(z)-\omega_{n+1}(\widehat{z})\right| \\
& \quad \leq \frac{1}{\Gamma(\alpha)} \int_{\widehat{z}, z}|z-\zeta|^{\alpha-1}\left|a(\zeta) \Phi\left(\omega_{n}(\psi(\zeta))\right)\right| \cdot|\mathrm{d} \zeta| \\
& \quad+\frac{1}{\Gamma(\alpha)} \int_{\widehat{z}, z}|z-\zeta|^{\alpha-1}|b(\zeta)| .|\mathrm{d} \zeta| \\
& \quad \leq \frac{\left(2+s_{2}\right)^{\alpha}}{\alpha \Gamma(\alpha)}\left(\|a\|_{\infty,[-1,1]^{k, s_{2}, n}}\|\Phi\|_{\infty,\left[-R_{0}, R_{0}\right]_{k, s_{2}, n}}+\|b\|_{\infty,[-1,1]^{k, s_{2}, n}}\right) \\
& \quad \cdot \varrho(z,[-1,1]) \\
& \quad \leq \Lambda\left(s_{2}\right) s_{2}(n+1)^{-(1 / k)} \\
& \quad<s_{2}(n+1)^{-(1 / k)} .
\end{aligned}
$$

Thence, the assertion $P(n+1)$ is true. Consequently, $P(n)$ is true for all $n \in \mathbb{N}^{*}$.

The proof of the proposition is then complete.

By virtue of the Proposition 2, we have for all $n \in \mathbb{N}^{*} \backslash\{1\}$ and $z \in[-1,1]^{k, s_{2}, n+1}$ 


$$
\begin{aligned}
\mid \omega_{n+1} & (z)-\omega_{n}(z) \mid \\
\leq & \frac{|z+1|^{\alpha}}{\Gamma(\alpha)} \int_{0}^{1}|1-s|^{\alpha-1}|a(-1+s(z+1))| \cdot \mid \Phi\left(\omega_{n}(\psi(-1\right. \\
& +s(z+1))))-\Phi\left(\omega_{n-1}(\psi(-1+s(z+1)))\right) \mid \mathrm{d} s \\
\leq & \frac{\left(2+s_{2}\right)^{\alpha}}{\alpha \Gamma(\alpha)}\|a\|_{\infty,[-1,1]^{k, s_{2}, n+1}}\left\|\Phi^{\prime}\right\|_{\infty,\left[-R_{0}, R_{0}\right]_{s_{2}, n+1}} \\
& \cdot\left\|\omega_{n}-\left(\omega_{\left.n-1 \mid[-1,1]^{k, s_{2}, n}\right)}\right)\right\|_{\infty,[-1,1]^{k, s_{2}, n}} \\
\leq & \Lambda\left(s_{2}\right)\left\|\omega_{n}-\left(\omega_{n-1 \mid[-1,1]^{k, s_{2}, n}}\right)\right\|_{\infty,[-1,1]^{k, s_{2}, n}}
\end{aligned}
$$

It follows that

$$
\begin{aligned}
& \left\|\omega_{n+1}-\left(\omega_{n \mid[-1,1]^{k, s_{2}, n+1}}\right)\right\|_{\infty,[-1,1]^{k, s_{2}, n+1}} \\
& \quad \leq \frac{\left\|\omega_{2}-\left(\omega_{1 \mid[-1,1]^{k, s_{2}, 2}}\right)\right\|_{\infty,[-1,1]^{k, s_{2}, 2}}}{\Lambda\left(s_{2}\right)} \Lambda\left(s_{2}\right)^{n}, \quad n \in \mathbb{N}^{*} \backslash\{1\} .
\end{aligned}
$$

Let us set $\Omega_{1}:=\omega_{1}$ and denote, for all $n \in \mathbb{N}^{*} \backslash\{1\}$, by $\Omega_{n}$ the function

$$
\begin{aligned}
\Omega_{n}:[-1,1]^{k, s_{2}, n+1} & \longrightarrow \mathbb{C}, \\
z & \longmapsto \omega_{n+1}(z)-\omega_{n}(z) .
\end{aligned}
$$

Then, the function $\Omega_{n}$ is holomorphic on $[-1,1]^{k, s_{2}, n+1}$ for each $n \in \mathbb{N}^{*}$. Furthermore, the following relations hold for every $n \in \mathbb{N}^{*} \backslash\{1\}$ :

$$
\left\{\begin{array}{l}
\left\|\Omega_{n}\right\|_{\infty,[-1,1]^{k, s_{2}, n+1}} \leq \frac{\left\|\omega_{2}-\left(\omega_{1 \mid[-1,1]^{k, s_{2}, 1}}\right)\right\|_{\infty,[-1,1]^{k, s_{2}, 1}}}{\Lambda\left(s_{2}\right)}\left(\Lambda\left(s_{2}\right)\right)^{n}, \\
\Omega_{n \mid[d, 1]}=f_{n-1} .
\end{array}\right.
$$

Since $\Lambda\left(s_{2}\right) \in[0,1[$, it follows then from (59) that the function series $\sum \Omega_{n \mid[-1,1]}$ is uniformly convergent on $[-1,1]$ to the function $u$. However, we know, according to relation (4) of Remark 1, that the following inclusion hold:

$$
[d, 1]_{k, s_{2}, n} \subset[-1,1]^{k, s_{2}, n}, \quad n \in \mathbb{N}^{*} .
$$

It follows then that

$$
\begin{cases}\left\|\Omega_{n}\right\|_{\infty,[d, 1]_{k, s_{2}, n}} \leq \frac{\left\|\omega_{2}-\left(\omega_{1 \mid[-1,1]^{k, s_{2}, n}}\right)\right\|_{\infty,[-1,1]^{k, s_{2}, 2}}}{\Lambda\left(s_{2}\right)}\left(\Lambda\left(s_{2}\right)\right)^{n}, & n \in \mathbb{N}^{*} \backslash\{1\}, \\ \Omega_{n \mid[d, 1]}=f_{n-1}, & n \in \mathbb{N}^{*} \backslash\{1\} .\end{cases}
$$

The relations (61) entail, thanks to the main result of [41], that $u_{\mid[d, 1]}$ belongs to $G_{k}([d, 1])$, for each $d \in$ ] $-1,1$ [. Thence, since $u$ is of class $C^{1}$ on $[-1,1]$, it follows that $u$ belongs to the Gevrey class $G_{k,-1}([-1,1])$.

The proof of the main result is then complete.

\section{Examples}

To obtain examples illustrating our main result, we need first to prove the following proposition.

Proposition 3. The function

$$
\begin{aligned}
\mathscr{L}: & \mathbb{C} \longrightarrow \mathbb{C}, \\
& z \longmapsto 2 e^{(z-1) / 2}-1,
\end{aligned}
$$

satisfies the property $S(l)$ for every $l \in] 0,1]$.

Proof. Let $l \in] 0,1], \varepsilon \in] 0,1]$ and $z \in[-1,1]^{\varepsilon}$. We have

$$
\mathscr{L}(z)=2 e^{(z-1) / 2}-1 .
$$

It follows that

$$
\operatorname{Re}(\mathscr{L}(z)+1)>0 .
$$

We consider then the principal argument $\arg (\mathscr{L}(z)+1)$ of $\mathscr{L}(z)+1$ which satisfies the following estimates:

$$
|\arg (\mathscr{L}(z)+1)|=\frac{|\operatorname{Im}(z)|}{2} \leq\left(1+\frac{\varepsilon}{2}\right) \tan \varepsilon .
$$

But, direct computations prove that

$$
0<\frac{\tan \varepsilon-\left(\varepsilon+\left(\varepsilon^{3} / 3\right)\right)}{\varepsilon^{3}}<\tan 1-\frac{4}{3} .
$$

Thence, we have

$$
\begin{aligned}
\left(1+\frac{\varepsilon}{2}\right) \tan \varepsilon & \leq\left(1+\frac{\varepsilon}{2}\right)\left(\varepsilon+\frac{\varepsilon^{3}}{3}+\left(\tan 1-\frac{4}{3}\right) \varepsilon^{3}\right) \\
& \leq \varepsilon+\left(\frac{3}{2} \tan 1-1\right) \varepsilon^{2} .
\end{aligned}
$$

It follows that

$$
\arg (\mathscr{L}(z)+1) \leq \varepsilon+\left(\frac{3}{2} \tan 1-1\right) \varepsilon^{2} .
$$

On the other hand, we have 


$$
\begin{aligned}
|\mathscr{L}(z)+1| & =2 e^{(\operatorname{Re}(z)-1) / 2} \\
& \leq 2 e^{\varepsilon / 2} .
\end{aligned}
$$

But, we know that

$$
\begin{aligned}
2 e^{\varepsilon / 2} & =2+\varepsilon+\varepsilon^{2} \sum_{n=0}^{+\infty} \frac{\varepsilon^{n}}{(n+2) ! 2^{n+2}} \\
& \leq 2+\varepsilon+\left(\sqrt{e}-\frac{3}{2}\right) \varepsilon^{2} .
\end{aligned}
$$

It follows that

$$
|\mathscr{L}(z)+1| \leq 2+\varepsilon+\left(\sqrt{e}-\frac{3}{2}\right) \varepsilon^{2} .
$$

We derive, from the estimates (68) and (71), the following inclusion:

$$
\mathscr{L}\left([-1,1]^{\varepsilon}\right) \subset[-1,1]^{\varepsilon+\mu \varepsilon^{2}},
$$

where

$$
\begin{aligned}
\mu & :=\max \left(\frac{3}{2} \tan 1-1, \sqrt{e}-\frac{3}{2}\right) \\
& =\frac{3}{2} \tan 1-1>0 .
\end{aligned}
$$

Let $n \in \mathbb{N}^{*}$ and $\left.A \in\right] 0,1 / \mu l[$. We have

$$
\begin{aligned}
& A(n+1)^{-1 / l}+\mu A^{2}(n+1)^{-2 / l}-A n^{-1 / l} \\
& =A n^{-1 / l}\left(\left(1+\frac{1}{n}\right)^{-1 / l}+\mu A \frac{n^{1 / l}}{(n+1)^{2 / l}}-1\right) \\
& \quad \leq A n^{-1 / l}\left(\left(1+\frac{1}{n}\right)^{-1 / l}+\frac{\mu A}{n}-1\right) .
\end{aligned}
$$

But, we have

$$
\left(1+\frac{1}{n}\right)^{-1 / l}+\frac{\mu A}{n}-1 \underset{n \longrightarrow+\infty}{\sim} \frac{\mu(A-(1 / \mu l))}{n} .
$$

It follows that there exists an integer $N_{A, l} \geq 1$ such that the following inequality holds for every integer $n \geq N_{A, l}$ :

$$
A(n+1)^{-1 / l}+\mu A^{2}(n+1)^{-2 / l} \leq A n^{-1 / l} .
$$

Consequently, we have

$$
\mathscr{L}\left([-1,1]^{A(n+1)^{-1 / l}}\right) \subset[-1,1]^{A n^{-1 / l}}, \quad n \geq N_{A, l},
$$

$$
\mathscr{L}\left([-1,1]^{l, A, n+1}\right) \subset[-1,1]^{l, A, n}, \quad n \geq N_{A, l} .
$$

It follows that the function satisfies the property $S(l)$. The proof of the proposition is then complete.

Recall that the following estimate holds for every $z \in \mathbb{C}$

$$
\max (|\sin (z)|,|\cos (z)|) \leq e^{|z|} .
$$

It means that the functions $\Phi_{1}:=\sin$ and $\Phi_{2}:=\cos$ satisfy the estimates:

$$
\left|\Phi_{j}(z)\right| \leq \alpha_{0} e^{\beta_{0}|z|}, \quad z \in \mathbb{C}, j=1,2,
$$

with $\alpha_{0}=\beta_{0}=1$.

Example 1. Let $C \in \mathbb{C}$ and $\gamma \in]-1,1[$. We assume that

$$
0<|C|<\min \left(\frac{\alpha \Gamma(\alpha)}{2^{\alpha+1} e^{\left(2^{\alpha} / \alpha \Gamma(\alpha)\right)|\gamma|+1}}, \frac{1-|\gamma|}{2}\right) .
$$

Consider the FFDE

$$
\left(E^{1}\right):{ }^{c} D_{-1}^{\alpha} f(x)=C(x+1) \sin \left(f\left(2 e^{(x-1) / 2}-1\right)\right)+\gamma \sin (x+1)
$$

with the initial condition

$$
\left(E_{1}^{1}\right): f(-1)=0 .
$$

Consider then the following entire functions:

$$
\left\{\begin{array}{l}
\Phi_{1}: z \longmapsto \sin (z), \\
a_{1}: z \longmapsto C(x+1), \\
b_{1}: z \longmapsto \gamma \sin (z+1) .
\end{array}\right.
$$

It is clear that $a_{1}$ is not identically vanishing and that $a_{1}(-1)=b_{1}(-1)=0$. Furthermore, we have

$$
\begin{aligned}
\frac{2^{\alpha}}{\alpha \Gamma(\alpha)}\left\|b_{1}\right\|_{\infty,[-1,1]}+|0| & =\frac{2^{\alpha}}{\alpha \Gamma(\alpha)}|\gamma| \\
& <\ln \left(\frac{\alpha \Gamma(\alpha)}{e 2^{\alpha+1}|C|}\right) \\
& =\frac{\ln \left(\alpha \Gamma(\alpha) / e \alpha_{0} \beta_{0} 2^{\alpha}\left\|a_{1}\right\|_{\infty,[-1,1]}\right)}{\beta_{0}} .
\end{aligned}
$$

We also have

that is,

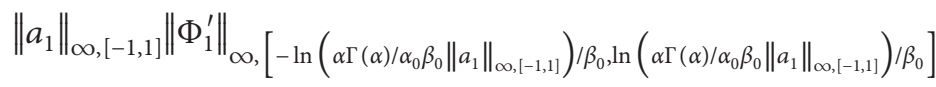

$$
\begin{aligned}
& \leq 2|C|<\frac{\alpha \Gamma(\alpha)}{2^{\alpha}},
\end{aligned}
$$

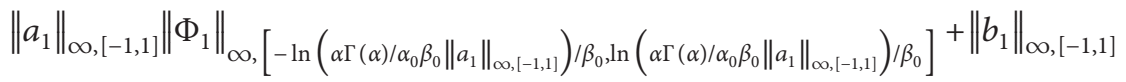

$$
\begin{aligned}
& \leq 2|C|+|\gamma|<1 \text {. }
\end{aligned}
$$


Consequently, it follows from the main result that the problem $\left(E^{1}\right)-\left(E_{1}^{1}\right)$ has a solution which belongs to the Gevrey class $G_{1,-1}([-1,1])$.

Example 2. Let $\eta>0$ and $\lambda \in \mathbb{C}$. We assume that

$$
\eta<\min \left(\frac{\alpha \Gamma(\alpha)}{2^{\alpha} e^{|\lambda|+1}}, 1\right) .
$$

Consider the FFDE

$$
\left(E^{2}\right):{ }^{c} D_{-1}^{\alpha} f(x)=\eta \sin (x+1) \cos \left(f\left(2 e^{(x-1) / 2}-1\right)\right)
$$

with the initial condition

$$
\left(E_{1}^{2}\right): f(-1)=\lambda .
$$

Consider then the following functions:

$$
\left\{\begin{array}{l}
\Phi_{2}: z \longmapsto \cos z, \\
a_{2}: z \longmapsto \eta \sin (z+1), \\
b_{2}: z \longmapsto 0 .
\end{array}\right.
$$

It is clear that $a_{2}$ is not identically vanishing and that $a_{2}(-1)=b_{2}(-1)=0$. Furthermore, we have the following inequalities:

$$
\begin{aligned}
& \frac{2^{\alpha}}{\alpha \Gamma(\alpha)}\left\|b_{2}\right\|_{\infty,[-1,1]}+|\lambda|=\ln \left(\frac{\alpha \Gamma(\alpha)}{e 2^{\alpha} \eta}\right)<\frac{\ln \left(\alpha \Gamma(\alpha) / e \alpha_{0} \beta_{0} 2^{\alpha}\left\|a_{2}\right\|_{\infty,[-1,1]}\right)}{\beta_{0}}, \\
& \left\|a_{2}\right\|_{\infty,[-1,1]}\left\|\Phi_{2}^{\prime}\right\|_{\infty,\left[-\left(\ln \left(\alpha \Gamma(\alpha) / \alpha_{0} \beta_{0}\left\|a_{1}\right\|_{\infty,[-1,1]}\right) / \beta_{0},\right)\left(\ln \left(\alpha \Gamma(\alpha) / \alpha_{0} \beta_{0}\left\|a_{1}\right\|_{\infty,[-1,1]}\right) / \beta_{0}\right)\right]} \leq \eta<\frac{\alpha \Gamma(\alpha)}{2^{\alpha}}, \\
& \left\|a_{2}\right\|_{\infty,[-1,1]}\left\|\Phi_{2}\right\|_{\infty,\left[-\left(\ln \left(\alpha \Gamma(\alpha) / \alpha_{0} \beta_{0}\left\|a_{1}\right\|_{\infty,[-1,1]}\right) / \beta_{0}\right),\left(\ln \left(\alpha \Gamma(\alpha) / \alpha_{0} \beta_{0}\left\|a_{1}\right\|_{\infty,[-1,1]}\right) / \beta_{0}\right)\right]}+\left\|b_{2}\right\|_{\infty,[-1,1]}=\eta<1 .
\end{aligned}
$$

Consequently, it follows from the main result that the problem $\left(E^{2}\right)-\left(E_{1}^{2}\right)$ has a solution which belongs to the Gevrey class $G_{1,-1}([-1,1])$.

\section{Data Availability}

No data were used to support this study.

\section{Disclosure}

This modest work is dedicated to the memories of two great men: our beloved master Ahmed Intissar (1951-2017), a brilliant mathematician (PhD at M.I.T, Cambridge), a distinguished professor, a man with a golden heart; our brother and indeed friend Mohamed Saber Bensaid (1965-2019), the man who belongs to the time of jasmine and sincere love, the comrade who devoted his whole life to the fight for socialism, democracy, and human rights.

\section{Conflicts of Interest}

The author declares that there are no conflicts of interest.

\section{References}

[1] K. B. Oldham and J. Spanier, The Fractional Calculus: Theory and Applications of Differentiation and Integration to Arbitrary Order, Academic Press, Chicago, IL, USA, 15th edition, 1974.

[2] R. L. Bagley and P. J. Torvik, "On the fractional calculus model of viscoelastic behavior," Journal of Rheology, vol. 30, no. 1, pp. 133-155, 1986.

[3] D. Baleanu, K. Diethelm, E. Scalas, and J. J. Trujillo, Fractional Calculus: Models and Numerical Methods, World Scientific, Singapore, 2nd edition, 2016.
[4] D. Baleanu, A. C. J. Luo, and J. A. T. Machado, Fractional Dynamics and Control, Springer, Berlin, Germany, 2012.

[5] F. Barpi and S. Valente, "Creep and fracture in concrete: a fractional order rate approach," Engineering Fracture Mechanics, vol. 70, no. 5, pp. 611-623, 2002.

[6] D. Craiem, F. J. Rojo, J. M. Atienza, G. V. Guinea, and R. L. Armentano, "Fractional calculus applied to model arterial viscoelasticity," Latin American Applied Research, vol. 38, no. 2, pp. 141-145, 2008.

[7] V. D. Djordjević, J. Jarić, B. Fabry, J. J. Fredberg, and D. Stamenović, "Fractional derivatives embody essential features of cell rheological behavior," Annals of Biomedical Engineering, vol. 31, no. 6, pp. 692-699, 2003.

[8] G. Jumarie, "Path integral for the probability of the trajectories generated by fractional dynamics subject to Gaussian white noise," Applied Mathematics Letters, vol. 20, no. 8, pp. 846-852, 2007.

[9] C. G. Koh and J. M. Kelly, "Application of fractional derivatives to seismic analysis of base-isolated models," Earthquake Engineering \& Structural Dynamics, vol. 19, no. 2, pp. 229-241, 1990.

[10] V. Lakshmikantham, "Theory of fractional functional differential equations," Nonlinear Analysis: Theory, Methods \& Applications, vol. 69, no. 10, pp. 3337-3343, 2008.

[11] Y. Liu, P. Lu, and I. Szanto, "Numerical analysis for a fractional differential time-delay model of HIV infection of $\mathrm{CD} 4^{+} \mathrm{T}$-cell proliferation under antiretroviral therapy," Abstract and Applied Analysis, vol. 2014, Article ID 291614, 13 pages, 2014.

[12] R. L. Magin, Fractional Calculus in Bioengineering, Begell House, Redding, CT, USA, 2006.

[13] F. Mainardi and P. Paradisi, "A model of diffusive waves in viscoelasticity based on fractional calculus," in Proceedings of the 36th IEEE Conference on Decision and Control, vol. 5, pp. 4961-4966, IEEE, San Diego, CA, USA, December 1997.

[14] F. Mainardi and G. Spada, "Creep, relaxation and viscosity properties for basic fractional models in rheology," The 
European Physical Journal Special Topics, vol. 193, no. 1, pp. 133-160, 2011.

[15] B. F. Martínez-Salgado, R. Rosas-Sampayo, A. TorresHernández, and C. Fuentes, "Application of fractional calculus to oil industry," in Fractal Analysis-Applications in Physics, Engineering and Technology, IntechOpen, London, UK, 2017.

[16] A. Mondol, R. Gupta, S. Das, and T. Dutta, "An insight into Newton's cooling law using fractional calculus," Journal of Applied Physics, vol. 123, no. 6, Article ID 064901, 2018.

[17] A. D. Myshkis and V. Kolmanovskii, Introduction To the Theory of Functional Differential Equations, Mathematics and its Applications, Springer, Berlin, Germany, 1999.

[18] R. Pakhira, U. Ghosh, and S. Sarkar, "Study of memory effects in an inventory model using fractional calculus," Applied Mathematical Sciences, vol. 12, no. 17, pp. 797-824, 2018.

[19] B. Ross, "Fractional Calculus and its Applications," in Lecture Notes in Mathematics, Springer, Berlin, Germany, 1975.

[20] S. Sengupta, U. Ghosh, S. Sarkar, and S. Das, "Application of fractional calculus to distinguish left ventricular hypertrophy with normal ECG," in Proceedings of the 2018 4th International Conference on Recent Advances in Information Technology (RAIT), pp. 1-6, IEEE, Dhanbad, India, March 2018.

[21] N. Shimizu and W. Zhang, "Fractional calculus approach to dynamic problems of viscoelastic materials," JSME International Journal Series C Mechanical Systems, Machine Elements and Manufacturing, vol. 42, no. 4, pp. 825-837, 1999.

[22] J. Wang and Y. Zhang, "Designing synchronization schemes for chaotic fractional-order unified systems," Chaos, Solitons \& Fractals, vol. 30, no. 5, pp. 1265-1272, 2006.

[23] B. Ross, An Introduction to Fractional Calculus and Fractional Differential Equations, A Wiley-Interscience Publication, Hoboken, NJ, USA, 1993.

[24] R. P. Agarwal, D. Baleanu, J. J. Nieto, D. F. M. Torres, and Y. Zhou, "A survey on fuzzy fractional differential and optimal control nonlocal evolution equations," Journal of Computational and Applied Mathematics, vol. 339, pp. 3-29, 2018.

[25] K. Diethelem, The Analysis of Fractional Functional Equations, Springer, Berlin, Germany, 2004.

[26] K. Diethelem and N. J. Ford, "Analysis of fractional differential equations," Journal of Mathematical Analysis and Its Applications, vol. 256, pp. 229-248, 2002.

[27] X.-L. Ding and J. J. Nieto, "Analytical solutions for multi-term time-space fractional partial differential equations with nonlocal damping terms," Fractional Calculus and Applied Analysis, vol. 21, no. 2, pp. 312-335, 2018.

[28] I. Podlubny, Fractional Differential Equations, Academic Press, Cambridge, MA, USA, 1999.

[29] M. Benchohra, J. Henderson, S. K. Ntouyas, and A. Ouahab, "Existence results for fractional order functional differential equations with infinite delay," Journal of Mathematical Analysis and Applications, vol. 338, no. 2, pp. 1340-1350, 2008.

[30] Y.-K. Chang, M. Mallika Arjunan, G. M. N’Guérékata, and V. Kavitha, "On global solutions to fractional functional differential equations with infinite delay in Fréchet spaces," Computers \& Mathematics with Applications, vol. 62, no. 3, pp. 1228-1237, 2011.

[31] J. Sousa, E. Capelas de Oliveira, and F. G. Rodrigues, "Stability of fractional functional differential equations," 2018, https:// arxiv.org/abs/1807.06145.
[32] N. V. Azbelev, V. P. Maksimov, and L. F. Rakhmtullina, Introduction to the Theory of Functional Differential Equations, Methods and Applications, Hindawi Publishing Corporation, London, UK, 2007.

[33] R. D. Driver, Ordinary and Delay Differential Equations, Applied Mathematical Sciences, Vol. 20, Springer, Berlin, Germany, 1977.

[34] J. Hale, Theory of Functional Differential Equations, Applied Mathematical Sciences, Vol. 3, Springer, Berlin, Germany, 1977.

[35] S. Bhalekar, V. Daftardar-Gejji, D. Baleanu, and R. Magin, "Fractional Bloch equation with delay," Computers \& Mathematics with Applications, vol. 61, no. 5, pp. 1355-1365, 2011.

[36] V. G. Pimenov and A. S. Hendy, "Numerical studies for fractional functional differential equations with delay based on BDF-type shifted Chebyshev approximations," Abstract and Applied Analysis, vol. 2015, Article ID 510875, 12 pages, 2015.

[37] G. Araújo, "Regularity and solvability of linear differential operators in Gevrey spaces," Mathematische Nachrichten, vol. 291, no. 5-6, pp. 729-758, 2018.

[38] G. Camliyurt, I. Kukavica, and V. Vicol, "Gevrey regularity for the Navier-Stokes in a half-space," Journal of Differential Equations, vol. 265, no. 9, pp. 4052-4075, 2018.

[39] J. Holmes, "Well-posedness and regularity of the generalized Burgers equation in periodic Gevrey spaces," Journal of Mathematical Analysis and Applications, vol. 454, no. 1, pp. 18-40, 2017.

[40] C. Foias and R. Temam, "Gevrey class regularity for the solutions of the Navier-Stokes equations," Journal of Functional Analysis, vol. 87, no. 2, pp. 359-369, 1989.

[41] E. Bendib and H. Zoubeir, "Développement en série de fonctions holomorphes des fonctions d'une classe de Gevrey sur l'intervalle [-1, 1]," Publications de l'Institut Mathématique (Belgrade), vol. 98, no. 112, pp. 287-293, 2015. 